\title{
LHomme
}

L'HOMME Revue française d'anthropologie

$213 \mid 2015$

Varia

\section{Dévoiler le travail de la mémoire de l'esclavage}

\section{Bogumil Jewsiewicki}

\section{OpenEdition}

\section{Journals}

Édition électronique

URL : http://journals.openedition.org/lhomme/23701

DOI : 10.4000//homme.23701

ISSN : 1953-8103

\section{Éditeur}

Éditions de l'EHESS

\section{Édition imprimée}

Date de publication : 26 février 2015

Pagination : 147-153

ISSN : 0439-4216

\section{Référence électronique}

Bogumil Jewsiewicki, « Dévoiler le travail de la mémoire de l'esclavage », L'Homme [En ligne],

213 | 2015, mis en ligne le 25 février 2017, consulté le 22 avril 2019. URL : http://

journals.openedition.org/lhomme/23701 ; DOI : 10.4000/lhomme.23701

(ㄷ École des hautes études en sciences sociales 


\section{Dévoiler le travail de la mémoire de l'esclavage}

\section{Bogumil Jewsiewicki}

L

E LIVRE DE Christine Chivallon, L'Esclavage, du souvenir à la mémoire, dont le sous-titre Contribution à une anthropologie de la Caraïbe est particulièrement prometteur, s'organise comme un trajet temporel: à partir des réalités mémorielles du présent pressenties comme illégitimes, le lecteur est renvoyé dans le passé pour revenir à un présent désormais transformé par l'analyse ethnohistorique opérée par l'auteure. Il se divise en deux blocs, d'environ 200 pages chacun, respectivement consacrés aux aspects théoriques et méthodologiques de la recherche sur la mémoire de l'esclavage, ainsi qu'aux modalités de présence/absence de cette mémoire dans la société martiniquaise. Alors que la première partie procède à l'examen critique et croisé de presque tout ce qui a été récemment publié en français et en anglais sur ces questions, la seconde applique le dispositif méthodologique ainsi élaboré pour identifier l'objet du souvenir, suivre le travail de la mémoire, surtout "incorporée », et accompagner les témoins, acteurs de la "fabrique mémorielle ». Pour le non-spécialiste de la Caraïbe que je suis, cet ouvrage apporte un éclairage précieux sur la question du travail de la mémoire dans des sociétés où l'identité collective a été marquée par un traumatisme fondateur, comme c'est le cas des sociétés post-esclavagistes et postcoloniales - les secondes ayant souvent succédé aux premières. Au moment de la transition entre un système organisé autour d'empires coloniaux et l'univers politique des États-nations, les représentations du passé, surtout fondées sur les documents écrits, ont légitimé le nouvel ordre politique. Ainsi l'histoire a-t-elle doté la nation d'un passé antérieur à la formation de l'État moderne, ce qui est contraire

À propos de Christine Chivallon, L'Esclavage, du souvenir à la mémoire. Contribution à une anthropologie de la Caraïbe, Paris, Karthala-CIRESC, 2012 («Esclavages»). 
à la chronologie du processus politique. Plus tard, l'entrée dans le système politique mondial des États-nations formés pendant la décolonisation a conduit au déplacement de l'instance de légitimation du récit. L'équivalence entre l'archive écrite et l'archive orale est alors reconnue mais sans que l'institution politique ne cède son droit de regard sur la provenance de l'information. Construite à partir de cette base, l'histoire continue à légitimer les rapports entre citoyen, nation et État. Depuis peu, alors qu'en nombre grandissant les citoyens accèdent à l'exercice effectif des droits politiques, les représentations du passé et l'autorité sur l'archive deviennent des enjeux politiques. L'histoire ayant perdu le monopole de la représentation légitime du passé collectif, le recours à la mémoire semblait répondre au besoin du pluralisme, comme le soutenait Pierre Nora pour la France. Il n'est pourtant pas avéré que le pluralisme des représentations mémorielles du passé instaure la reconnaissance effective de l'égalité citoyenne face aux institutions de l'État et, surtout, face à l'accès aux ressources que ce dernier réglemente.

Christine Chivallon intervient dans ce débat à partir d'un maillage entre la théorie du social, sensible aux effets politique du discours savant, et une enquête empirique réalisée à la Martinique. Dans la formation des sociétés antillaises actuelles, la place de la traite atlantique puis de la colonisation a donné aux rapports de race un rôle comparable aux rapports de classe dans la formation des sociétés occidentales. En ce qui concerne les représentations du passé, les modalités de l'usage politique du rapport de race, allant de sa négation à l'affirmation de sa centralité, ont eu un profond impact sur la qualification de la relation du citoyen à l'État. Il faut en tenir compte pour comprendre le choix du titre de ce livre, L'Esclavage, du souvenir à la mémoire: de son instauration à son abolition, l'esclavage est l'élément référent - même s'il en est souvent explicitement absent des représentations du passé. Christine Chivallon montre bien qu'il n'est pas innocent que le récit collectif prenne pour point nodal soit l'abolition, soit la capture et la réduction au statut de marchandise humaine, ou encore la résistance tant collective qu'individuelle aux effets politiques de l'esclavage d'après l'abolition. Du choix de l'événement «fondateur » de la représentation du passé (Christine Chivallon parle de "scène primordiale »), dépendent évidemment le déroulement et la qualification du temps historique, la hiérarchie d'acteurs dont l'agencéité est reconnue et les modalités de la mise en représentation, incluant la désignation d'archives qui légitimeront le témoin. La mémoire incorporée assure la présence discrète mais permanente du traumatisme initial, le témoin rend cette expérience effective par l'intermédiaire des séquelles qu'il en a gardées, plutôt que par la verbalisation. Alors, la chaîne sociale de transmission se 
fait archive ; très judicieusement, une trentaine de pages du livre, portant sur le corpus généalogique, est consacrée à démontrer son authenticité : à partir de cette archive, s'effectue la "production du sens générée à partir de l'expérience historique, à savoir les modes de sa conscientisation, via le travail symbolique opéré sur le souvenir et son référent » (p. 167).

Pour comprendre ce travail qu'elle dévoile, Christine Chivallon mobilise donc un très vaste corpus de travaux théoriques et anthropologiques sur l'imaginaire, sur la mémoire et sur le souvenir de l'esclavage. Ainsi s'expliquent ses choix méthodologiques, en particulier sa préférence au «travail de la mémoire » de Paul Ricœur plutôt qu'aux "lieux de mémoire » de Pierre Nora. Puisqu'elle considère que le rapport de force ancestral irrigue les édifices sociaux, Christine Chivallon a privilégié l'enquête sur le nom et la terre (leur transmission). Cette démarche ouvre l'accès à des registres mémoriels autres que les récits manifestés et conduit à des lieux de déploiement non verbalisés du mémoriel.

La distinction qu'elle établit entre le souvenir et la mémoire est particulièrement importante pour saisir les enjeux de la reconnaissance du travail de la mémoire comme rapport légitime au passé. Dans les sociétés post-esclavagistes contemporaines, la brutale dépossession - comparable à la prolétarisation (l'auteure n'utilise pas ce terme) - est intervenue à deux reprises, à l'origine de leur formation sociale, puis entre l'abolition de l'esclavage et la décolonisation: la personne capturée en Afrique est devenue une marchandise dont la capacité de travail était la seule valeur, puis, à l'abolition, ses descendants ont plongé dans un interminable conflit pour le contrôle de cette capacité, sans que, pour la plupart, les affranchis n'aient jamais accédé à la terre. Depuis ses travaux antérieurs sur la Martinique, Christine Chivallon souligne l'importance politique et symbolique du rapport à la terre. Faute de «moyen d'accrocher les mémoires aux noms et aux lieux", il semble impossible de composer "la trame de récits réparateurs» (p. 530). Dans ce contexte, le passage du souvenir à la mémoire, en tant que modalité sociale du rapport au passé, est crucial pour le plein exercice des droits citoyens. Christine Chivallon montre que le souvenir se réfere à ce qui est advenu et recouvre le « réel » de l'événement. Pour obtenir la reconnaissance politique de «l'expérience fondatrice de la perte et de l'oppression ", il faut qu'interviennent sa transmission et sa mise en récit, il faut une mémoire historique au fondement de l'identité collective. Dans sa fine analyse du travail de la mémoire et de l'épistémologie du souvenir, Christine Chivallon met justement l'accent sur la place tenue par l'imaginaire, puisque le recours au système de signes contribue à instituer une vision du monde et un cadre social de la mémoire. Le va-et-vient entre les philosophes et théoriciens, d'une part, 
et les anthropologues, d'autre part, lui permet l'élaboration d'un dispositif méthodologique dont la pertinence dépasse le cadre de sa recherche. La formulation suivante traduit l'objectif de ce livre, mais elle a une portée nettement plus large :

«[...] rechercher dans les souvenirs relatés par les témoins, les manières de signifier les dimensions d'une existence placée sous le sceau de la répétition de chocs événementiels traumatiques au sein d'un contexte lui-même édifié sur le socle de la violence » (p. 168).

Lorsqu'elle examine la façon dont l'Insurrection du Sud, qui opposa en 1870 des anciens maîtres à des anciens esclaves, fut intégrée à l'expérience et l'identité collectives, Christine Chivallon suit «le trajet de souvenirs depuis la scène fondatrice qu'ils relatent jusqu'à leurs formalisations possibles en récits de mémoire» (p. 165). Lorsqu’elle considère séparément les souvenirs incorporés et la mémoire, distingue l'incorporation de l'historicisation, le «souvenir-empreinte » de la "mémoire-construite ", elle fait reposer la médiation sur la figure du témoin et sur sa sensibilité au passé. Le paysan martiniquais (sans attribution de genre), ou celui/celle qui aspire à l'être, est porteur de ces mémoires et témoin de l'actualité de l'expérience de l'univers esclavagiste. Elle explore les outils de la représentation pour remettre en question la "différentiation de l'intolérable " faisant que l'accès au recouvrement d'une dignité historique se distribue de façon différentielle. Elle accentue l'importance de la mémoire incorporée, relativise l'exigence de la verbalisation du souvenir afin qu'une mémoire soit reconnue dicible, visible et/ou audible, rappelle la place des pratiques corporelles relatives à la mémoire. En définitive, elle valorise d'autres formes de représentations que le récit pour rendre un passé présent et agissant, même si l'enjeu autour de la construction du récit national demeure.

Très critique envers l'épistémologie de la vérification, Christine Chivallon prend néanmoins le parti de Richard Price dans la controverse qui l'oppose à David Scott, tout en explicitant sa démarche. Analyser le dispositif narratif du souvenir de l'esclavage permet de discerner un récit identitaire instituant des modes d'être ensemble et installant un principe de permanence de soi tant individuel que collectif. Dans la Caraïbe, mais aussi ailleurs, les évocations du passé, conservées et transmises par des individus et groupes privés de présence dans l'espace public, ont été réduites au silence. Cette disqualification, largement opérée par des idéologies politiques prônant l'unité, la cohésion nationale (etc.), marque aussi le discours savant sur les mémoires minorées. À cette occasion, comme plusieurs spécialistes de la Caraïbe, Christine Chivallon convoque l'œuvre poétique de Derek Walcott, lue comme l'attestation de l'absence du souvenir de l'esclavage. 
Je ne partage pas cette lecture. Lorsque, dans son poème The Royal Palms... An Absence of Ruins, Walcott met en évidence l'absence de traces matérielles, il ne signifie pas le vide mémoriel, bien au contraire. Si les vestiges du passé des descendants d'esclaves ne sont pas apparents, c'est parce que le regard de l'histoire a planté dans son champ d'observation des ruines dans lesquelles ces derniers ne se reconnaissent pas : ils refusent d'accepter comme les leurs, les ruines d'une exploitation sucrière, lieu de mémoire d'expériences traumatiques ${ }^{1}$ et de la marginalisation qui s'ensuivit. Pour Walcott, l'histoire est écrite à partir d'un regard biaisé (Ann Stoler aurait écrit "disregard $»^{2}$ ) porté par les autres, tandis que l'expérience inscrite dans l'imaginaire ${ }^{3}$ est transmise en héritage par les sociétés antillaises. En insistant sur l'absence (relative) des ruines, il fait ressortir le caractère totalitaire de l'histoire ${ }^{4}$ : « History which looked over the shoulder of the engraver and, later, the photographer. History can alter the eye and the moving hand to conform a view of itself ». Ailleurs, il écrit " every island, is an effort of memory", et redit l'importance du travail de la mémoire pour contrer les effets de l'écriture historienne : "every racial biography culminating in amnesia and fog". Pour cette raison, il oppose l'art à l'histoire: "process of the making of poetry, or what should be called not its "making" but its remaking, the fragmented memory [...] art is this restoration of our shattered histories " 5 . A la place des ruines et des héros fabriqués par l'histoire, «I see cabbage palms moving their fronds at sunrise, I think they are reciting Perse [...]. At least islands not written about but writing themselves $! »^{6}$. La voix de Saint-John Perse, poète antillais qui reçut lui aussi le prix $\mathrm{Nobel}^{7}$, «make[s] out of these foresters and fishermen heraldic men $!{ }^{8}$. L'artiste plutôt que l'historien révèle

1. Walcott inscrit la violence au cœur de l'héritage antillais: «Decimation from the Aruac downwards is the blasted root of Antillean history ». Cette citation et, à moins d'une indication contraire, les suivantes sont tirées de la transcription du discours The Antilles. Fragments of Epic Memory, disponible sur : http://poetry.rapgenius.com/Derek-walcott-the-antilles-fragments-ofepic-memory-annotated. Le discours a été publié dans Sture Allén, ed., Nobel Lectures in Literature 1991-1995, Singapore, World Scientific Publishing Co, 1997.

2. Cf. le chapitre "Imperial Dispositions of Disregard", in Ann Stoler, Along the Archival Grain. Epistemic Anxieties and Colonial Common Sense, Princeton, Princeton University Press, 2009.

3. Walcott rejette la distinction entre «imagination and collective memory».

4. "The history of the world, by which of course we mean Europe, is a record of intertribal lacerations, of ethnic cleansings " (cf. The Antilles. Fragments of Epic Memory).

5. «Break a vase, and the love that reassembles the fragments is stronger than that love which took its symmetry for granted when it was whole. The glue that fits the pieces is the sealing of its original shape " (Ibid.).

6. Extrait du poème What the Twilight Says, London, Faber \& Faber, 1998 : 78.

7. Saint-John Perse, de son vrai nom Alexis Leger, était né à Pointe-à-Pitre en Guadeloupe en 1887 ; il reçut le prix Nobel de littérature en 1960, trente-deux ans avant Derek Walcott.

8. Cf. Collected Poems 1948-1984, London-New York, Farrar, Straus \& Giroux, 1987 : 217. 
aux vivants l'expérience et le patrimoine ignorés par l'histoire: «[T]he past is the sculpture and the present the beads of dew or rain on the forehead of the past [...]. [T] he process of poetry is one of excavation and of self-discovery". Ainsi, "Caribbean culture is not evolving but already shaped. Its proportions are not to be measured by the traveller or the exile, but by its own citizenry and architecture $"$.

Or, contrairement à l'impression que pourrait laisser un jugement trop rapide, les positions de Walcott et de Chivallon s'avèrent très proches. Le référent esclavage n'a pas besoin d'être nommé pour que ses appropriations symboliques et les incorporations des expériences de ses conséquences organisent les univers de sens d'un groupe social. Le poids du dispositif de contrôle politique et social peut rendre contre-productive la transmission mémorielle de l'événement esclavage impliquant, par exemple, l'impératif de reconnaissance pour son abolition. En revanche, un travail de mémoire à partir de l'objet esclavage autorise la constitution d'une chaîne de transmission du souvenir sur l'archive alternative. Non verbalisé, le récit identitaire diachronique émerge de la mémoire incorporée qui se refuse d'obéir à l'autorité de l'histoire et à son injonction de soumettre le particulier à la discipline de l'universel. C'est plutôt, comme l'a dit Walcott, l'intensité de ce particulier qui lui confere une valeur universelle.

Christine Chivallon a donc concentré son attention, non plus sur le fait historique massif, c'est-à-dire l'esclavage en tant que ruine sous le contrôle de l'histoire, mais sur l'actualisation par procuration (vicarious) de son souvenir, à partir de laquelle ledit souvenir aide à construire le temps en amont et en aval de l'événement. Ainsi, plutôt que des matériaux pour une "mémoire à venir ", les souvenirs de l'insurrection de 1870 annulent la distance à son référent, la distance "entre une mémoire de l'esclavage et l'esclavage lui-même» (p. 299). L'impossible oubli de l'esclavage n'a d'équivalent dans le champ politique que dans le rejet de l'injonction de s'en approprier explicitement les ruines. Pour « reconstruire, restaurer et rétablir le lien social»(p. 399), le tissage des souvenirs de l'insurrection de 1870 est une modalité de la mémoire collective de l'esclavage dont la présence est ainsi réactivée dans l'événement dont les témoins, inscrits dans la chaîne de transmission sociale, maintiennent vivant le souvenir ${ }^{10}$.

9. Il convoque la tradition homérique (Omeros) autant que "the soundlike a rumour without any echo of History", "The Sea as History" (cf. le site "Selected Poems of Derek Walcott": http://fr.scribd.com/doc/79752975/Selected-poems-of-Derek-Walcott ). Ailleurs, il note: "The intensity of the particular is what makes it general " (in Natasha Saje \& George Handley, "Sharing in the Exhilaration : An Interview with Derek Walcott ", Ariel, 2001, 32 [2] : 135).

10. Christine Chivallon souligne que la portée mémorielle de cet événement (l'insurrection de 1870) ne peut être pleinement comprise qu'en le resituant dans le contexte "psychodramatique» de l'instauration de la Troisième République (pp. 400-401). 
Cette mémoire est médiatrice d'une continuité qui s'évertue à maintenir la permanence d'un corps collectif et à exiger la reconnaissance de sa dignité. Montrer que le travail de la mémoire ne s'effectue pas nécessairement sur l'événement traumatique fondateur mais plutôt sur un événement relais (délégué, vicarious) n'est pas le moindre des mérites de ce livre ${ }^{11}$. La minutie de l'enquête empirique et la finesse de l'analyse des données impressionnent autant que l'attention accordée aux témoins comme coproducteurs de l'agencement des processus mémoriels. Quelques réserves mineures ${ }^{12}$ ne m'empêchent pas de recommander ce tour de force à tout(e) chercheur(e) en quête d'une perspective théorique et d'un dispositif méthodologique permettant d'enquêter sur l'identité et la reconnaissance dans les sociétés contemporaines marquées par un traumatisme fondateur.

Université Laval

Département d'histoire, Québec (Canada)

Bogumil.Koss@hst.ulaval.ca

MOTS CLÉS/KEYWORDS : Caraïbes/Carribean - Martinique - esclavage/slavery - mémoire/ memory - souvenir/recall - récit/narrative - sociétés postcoloniales/postcolonial societies ethnohistoire/ ethnohistory - Derek Walcott.

11. Mes propres recherches en République démocratique du Congo m’ont permis de mettre en évidence un déplacement similaire, mais situant l'événement délégué en amont plutôt qu'en aval de l'événement fondateur. À travers l'enseignement scolaire de niveau élémentaire, en langues locales et dispensé par les missions chrétiennes, le pouvoir colonial belge a cherché à asseoir sa légitimité sur le souvenir de l'éradication de la traite orientale : le recours à la symbolique biblique lui a conféré la valeur de "sortie d'Égypte». Cependant, la mémoire collective congolaise a opéré un renversement des qualifications. Dans son discours à la cérémonie d'accession à la souveraineté nationale, le Premier ministre congolais, Patrice Lumumba, a qualifié la période coloniale d'esclavage. Puis, la peinture populaire urbaine, principal médium d'expression de la mémoire, présente Lumumba comme le Moïse des Congolais, esclaves de l'État colonial. Une autre icône de cette mémoire présente la peine de fouet administrée à un prisonnier sous l'œil d'un administrateur colonial. Le drapeau belge - jaune, noir et rouge - flotte au-dessus de la scène. Le prisonnier porte un maillot rayé noir et jaune alors que le fouet trace des lignes de sang rouges sur ses fesses nues. Le sujet colonial est propriété de l'État, son esclave. Cette image emprunte son organisation à la fameuse peinture Le Châtiment des quatre piquets dans les colonies de Marcel Verdier (1843), probablement via un tableau belge de la fin du XIXe siècle de Mandau intitulé "La civilisation au Congo ". Le référent esclavage façonné par les mises en signification tirées de la culture globale n'y conserve aucun lien avec la mémoire vive de l'esclavage interne aux sociétés congolaises. La traite des esclaves est un événement délégué pour activer le travail de la mémoire de l'asservissement par l'État colonial puis postcolonial.

12. Ces réserves sont ponctuelles, en voici quelques exemples. Comme tout binôme, et il n'est pas le seul dans le livre, le terme de contre-mémoire risque de produire l'impression d'opposition mécanique ce qui n'est sûrement pas l'intention de l'auteure. Sur un autre registre, malgré tout mon respect pour l'héritage intellectuel de Pierre Bourdieu, je ne suis pas convaincu par les efforts de Christine Chivallon d’appliquer son appareil conceptuel à l'analyse du travail de la mémoire. Puisque son érudition est très impressionnante, l'absence des travaux de Nathan Wachtel surprend d'autant plus. L'Invention du quotidien de Michel de Certeau est citée, mais je n'ai trouvé aucune mention de son concept opératoire de "propre", à mon avis très pertinent pour la démarche de l'auteure. J'estime également que le concept de « lieu de mémoire » de Pierre Nora est trop rapidement jugé inopérant pour sa recherche. 